\title{
Rapid quantification of microRNAs in plasma using a fast real-time PCR system
}

\author{
William John Andrews, Eoin Daniel Brown, Margaret Dellett, Ruth Esther Hogg, and David Arthur Simpson \\ Centre for Experimental Medicine, Queen's University Belfast, Belfast, Northern Ireland, United Kingdom
}

BioTechniques 58:244-252 (May 2015) doi 10.2144/000114287

Keywords: PCR; microRNA; RT-PCR; biomarker

Supplementary material for this article is available at www.BioTechniques.com/article/114287.

The ability to rapidly detect circulating small RNAs, in particular microRNAs (miRNAs), would further increase their already established potential as biomarkers for a range of conditions. One rate-limiting factor in miRNA detection is the time taken to perform quantitative real-time PCR (qPCR) amplification. We therefore evaluated the ability of a novel thermal cycler to perform this step in less than 10 minutes. Quantitative PCR was performed on an xxpress thermal cycler (BJS Biotechnologies), which employs a resistive heating system and forced air cooling to achieve thermal ramp rates of $10^{\circ} \mathrm{C} / \mathrm{s}$, and a conventional Peltier-controlled LightCycler 480 system (Roche) ramping at $4.8^{\circ} \mathrm{C} / \mathrm{s}$. The quantification cycle $\left(\mathrm{C}_{\mathrm{q}}\right)$ for detection of $18 \mathrm{~S}$ rDNA from a standard genomic DNA sample was significantly more variable across the block (F-test, $\left.P=2.4 \times 10^{-25}\right)$ for the xxpress $(20.01 \pm 0.47 \mathrm{SD})$ than for the LightCycler (19.87 \pm 0.04 SD). RNA was extracted from human plasma, reverse transcribed, and a panel of miRNAs was amplified and detected using SYBR Green. The sensitivities of the two systems were broadly comparable - both detected a panel of miRNAs reliably, and both indicated similar relative abundances. The xxpress thermal cycler facilitates rapid qPCR detection of small RNAs and brings point-of-care diagnostics based upon detection of circulating miRNAs a step closer to reality.

PCR is ubiquitous throughout the life and medical sciences, and a reduction in the time required to complete a PCR reaction would therefore be of immense benefit. While the choice of a fast enzyme is important for the optimization of fast PCR-based systems (1,2), the speed at which the temperature of the sample can be altered during thermal cycling is the primary rate-limiting factor (3). Using prototype systems, many investigators have demonstrated that rapid thermal cycling is possible $(4,5)$ and, under extreme conditions, can be completed in less than $1 \mathrm{~min}$ (6). The predominant format of existing thermal cyclers comprises a 96- or 384-well block, the temperature of which is controlled by a Peltier-based system limited to ramp rates of approximately $4^{\circ} \mathrm{C} / \mathrm{s}$. Several quantitative PCR systems that employ rapid thermal cycling are available commercially, but these are based on glass capillaries (LightCycler; Roche) (7) or plastic tubes placed in a centrifuge (Rotor-Gene; Qiagen). An alternative rapid plate-based approach, which can be more easily integrated into existing workflows, has now been developed. The xxpress thermal cycler (BJS Biotechnologies) employs resistive heating and forced air cooling to enable ramp rates of up to $10^{\circ} \mathrm{C} / \mathrm{s}$.

One of the main applications of PCR is for the quantitation of RNA targets. Following reverse transcription into cDNA, amplification of targets is detected by incorporation of a double- stranded DNA binding fluorescent dye (principally SYBR Green) or use of a sequence-specific probe-based system (e.g., TaqMan). This quantitative reverse transcription PCR (RT-qPCR) approach can be modified to measure small RNAs, specifically microRNAs (miRNAs). Following the discovery that miRNAs exist in a stable form within blood $(8,9)$, their potential as biomarkers was soon realized. miRNA expression patterns characteristic of cancer, cardiovascular disease, diabetes, Alzheimer's, and many other conditions have now been reported (10-12). Typically, global miRNA profiles are initially assessed in a discovery cohort using microarrays or deep sequencing, and selected informative miRNAs are subsequently

\section{METHOD SUMMARY}

Here we present a quantitative PCR platform that enables faster ramping between temperatures than conventional Peltier-based systems, thereby reducing the time required to complete a PCR reaction. This is particularly important for the development of clinical biomarkers for acute conditions, and we demonstrate the feasibility of this approach for detecting miRNAs in plasma. 
measured in a larger population. The method of choice for measuring a defined, diagnostic panel of miRNAs is RT-qPCR.

To perform RT-qPCR, RNA must be extracted from plasma or serum and reverse transcribed. Target miRNAs must then be amplified in individual PCR reactions. This process takes several hours and is sufficient for the current applications for detecting circulating miRNA biomarkers for which results may be reported within days or weeks. However, a more rapid assay would facilitate point-of-care diagnostics and expand potential uses. For example, changes in miRNAs have been associated with cardiac disease (10), and the ability to measure them in plasma quickly might provide earlier biomarkers for diagnosis of myocardial infarction and ensure more timely therapeutic intervention (13).

We therefore assessed the ability of the xxpress system (BJS Biotechnologies, Perivale, UK) to quantify miRNAs in plasma using high speed cycling in comparison with an existing qPCR system (LightCycler 480; Roche, Basel, Switzerland). It was possible to amplify RNAs from plasma cDNA in less than 10 min using the xxpress system, compared with 40 min with the LightCycler. However, although the performance of both systems was broadly comparable, variability across the plate and between replicate samples was greater in the xxpress.

\section{Materials and methods \\ Oligonucleotide synthesis}

All oligonucleotides (Integrated DNA Technologies, Leuven, Belgium) were reconstituted as $100 \mu \mathrm{M}$ stocks, DNA in TE buffer $(10 \mathrm{mM}$ Tris- $\mathrm{HCl}, 1 \mathrm{mM}$ EDTA, pH 8.0) and RNA in nuclease free water, and then stored at $-80^{\circ} \mathrm{C}$. Sequences of the RNA miRNA mimic, reverse transcription oligo, 18S primers, and miRNA primers are shown in Table 1.

\section{qPCR instruments}

The xxpress system (Figure 1) employs resistive heating and forced air cooling to facilitate rapid changes in temperature. PCR reactions are performed within proprietary "xxplates," which comprise a metal base fused with plastic
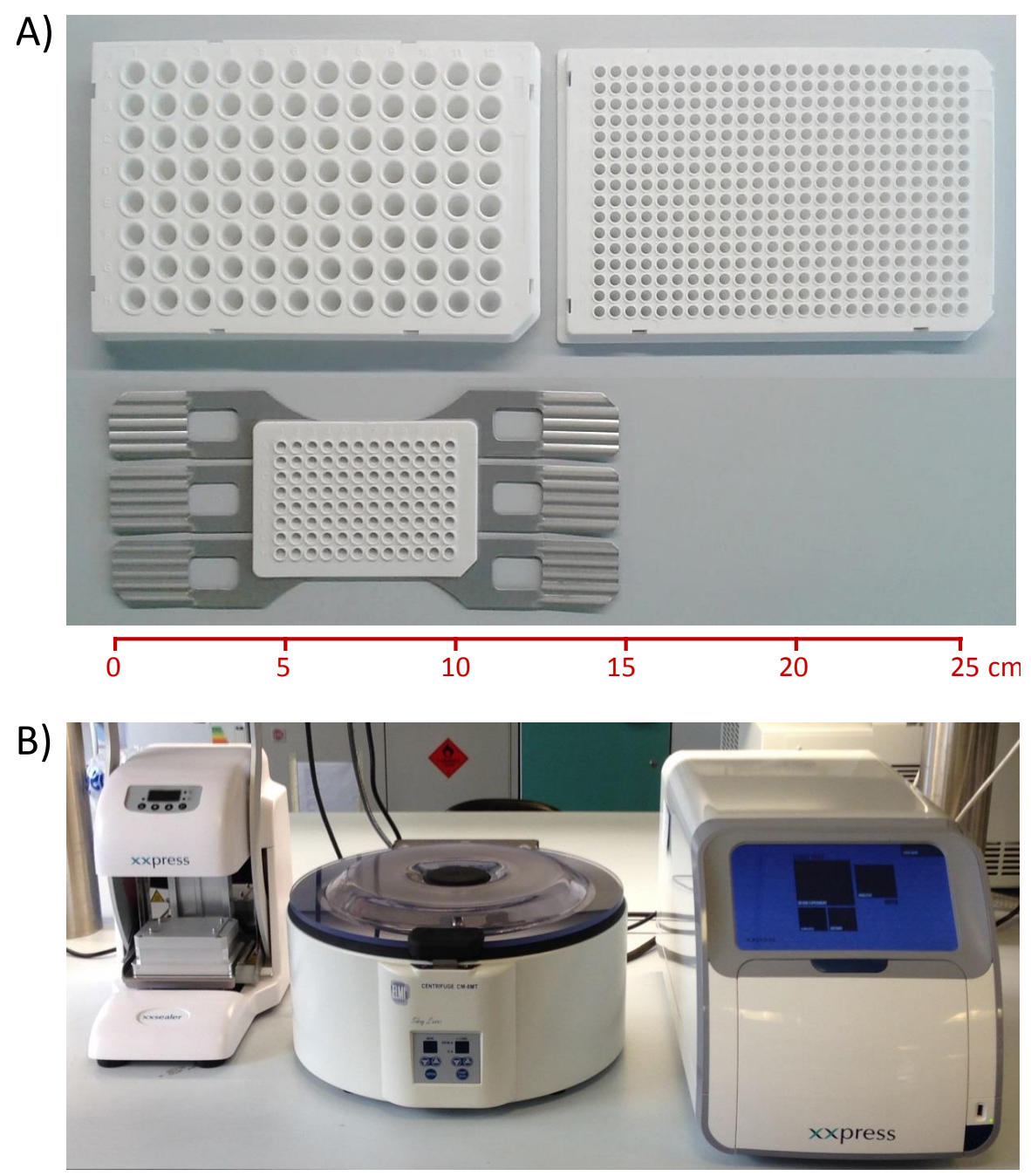

Figure 1. The xxpress system. (A) A 96-well xxplate (bottom) compared with conventional 96-well and 384-well PCR plates (upper). (Scale bar represents $25 \mathrm{~cm}$ to allow a direct size comparison.) (B) The xxpress system comprises a heat sealer (left), centrifuge (center), and cycler unit (right).

Table 1. Oligonucleotides.

\begin{tabular}{|c|c|c|c|}
\hline & Name & Sequence $\left(5^{\prime}-3^{\prime}\right)$ & $\mathrm{T}_{\mathrm{M}}\left({ }^{\circ} \mathbf{C}\right)$ \\
\hline \multirow[t]{3}{*}{ RT oligos } & miR-21 miRNA mimic & UAG CUU AUC AGA CUG AUG UUG AAA AAA AAA AAA A & 52.4 \\
\hline & OligodT-RACE & GCG AGC ACA GAA TTA ATA CGA CTC ACT ATA GGT TTT TTT TTT TTV N & 61.9 \\
\hline & Reverse RACE & GCG AGC ACA GAA TTA ATA CGA C & 53.9 \\
\hline \multirow[t]{2}{*}{$18 S$} & $18 \mathrm{~S}$ forward & AAA CGG CTA CCA CAT CCA AG & 55.3 \\
\hline & $18 S$ reverse & CCT CCA ATG GAT CCT CGT TA & 53.8 \\
\hline \multirow[t]{5}{*}{ miRNAs } & miR-10b-5p & TAC CCT GTA GAA CCG AAT TTG TG & 54.7 \\
\hline & miR-468-5p & TCC TGT ACT GAG CTG CCC CGA & 62.8 \\
\hline & miR-126-5p & CAT TAT TAC TTT TGG TAC GCG & 49.6 \\
\hline & miR-22-3p & AAG CTG CCA GTT GAA GAA CTG T & 57.1 \\
\hline & $\operatorname{miR}-21-5 p$ & TAG CTT ATC AGA CTG ATG TTG A & 50.9 \\
\hline \multirow[t]{2}{*}{ Y-RNAs } & Y-RNA-3p & CCC CCA CTG CTA AAT TTG ACT & 55.2 \\
\hline & Y-RNA-5p & GGC TGG TCC GAT GGT AGT & 56.8 \\
\hline \multicolumn{4}{|c|}{$\begin{array}{l}\text { Sequence and melting temperatures for oligonucleotides used in this investigation. Column } 1 \text { denotes } \\
\text { the general class of oligonucleotides used, and Column } 2 \text { denotes the name of each, also providing } \\
\text { information about target strand }(5 p \text { or } 3 p \text { for miRNAs). Column } 3 \text { provides sequence information } \\
\text { ( } V \text { denotes any nucleotide not } T \text { or } U \text {, and } N \text { denotes any nucleotide). Column } 4 \mathrm{~T}_{\mathrm{M}} \text { values were } \\
\text { calculated by Integrated DNA Technologies. }\end{array}$} \\
\hline
\end{tabular}




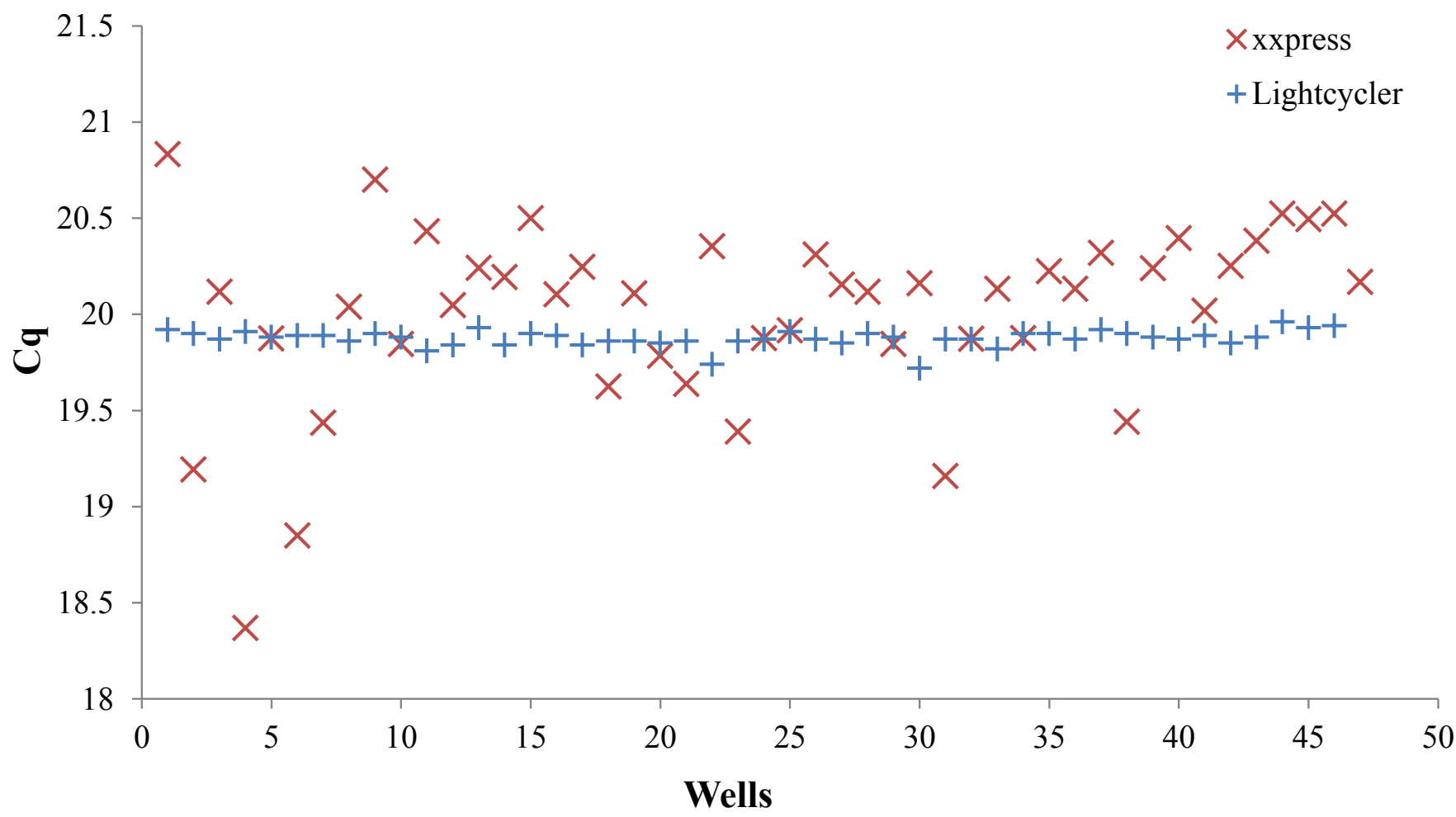

Figure 2. Variability in $\mathbf{C}_{\mathrm{q}}$ values across plates on both systems. A single $18 \mathrm{~S}$ rDNA PCR mastermix was distributed across 48 wells of both a Lightcycler 384 -well plate and an xxpress 96 -well xxplate ( $5 \mu \mathrm{L}$ reaction/well). PCR was performed with the same ramp rate on each thermal cycler $\left(4.8^{\circ} \mathrm{C} / \mathrm{s}\right)$. $\mathrm{C}_{\mathrm{q}}$ values are presented by row from well A1 (active wells are illustrated in Supplementary Figure S1). Melting curve analysis was performed to confirm amplification of a single product (data not shown).

wells, meaning that the sample is only $10 \mu \mathrm{m}$ from the heat source (Figure 1A). To enable thermal uniformity across all of the samples to within $\pm 0.3^{\circ} \mathrm{C}$ during holds at a static temperature or within $\pm 0.8^{\circ} \mathrm{C}$ during fast ramping $\left(10^{\circ} \mathrm{C} / \mathrm{s}\right)$, the xxpress uses an array of infrared sensors to determine the temperature of the test samples, and a control algorithm adjusts the heating patterns at a rate of 100 times per second. The xxplates to be analyzed using the xxpress system were prepared as follows: Plates were sealed using xxpress compatible polarized sealing strips heated at $170^{\circ} \mathrm{C}$ for $1.5 \mathrm{~s}$ using the heat sealer provided (Figure 1B). They were then centrifuged at 1000 rpm for $1 \mathrm{~min}$ in dedicated holders in the centrifuge provided before loading into the xxpress cycling unit (Figure 1B). The LightCycler 480 system with a 384-well block installed has a thermal uniformity of $\pm 0.4^{\circ} \mathrm{C}$ within $60 \mathrm{~s}$ of target attainment $\left(72^{\circ} \mathrm{C}\right)$. The PCR plates (Roche) to be analyzed were prepared following the manufacturer's instructions (LightCycler 480 user manual). In brief, plates were sealed using the manufacturer's sealing strips, ensuring all wells were securely covered, followed by centrifugation at 1200 rpm for 2 min. Plates were then loaded into the LightCycler.

PCR conditions and data analysis All PCR reactions were performed for 40 cycles with SYBR Fast qPCR

Table 2. PCR cycling conditions.

\begin{tabular}{|c|c|c|c|c|c|c|}
\hline & \multicolumn{3}{|c|}{ xxpress } & \multicolumn{3}{|c|}{ LightCycler 480} \\
\hline & Temp $\left({ }^{\circ} \mathbf{C}\right)$ & Hold (s) & Ramp rate $\left({ }^{\circ} \mathbf{C} / \mathbf{s}\right)$ & Temp $\left({ }^{\circ} \mathbf{C}\right)$ & Hold (s) & Ramp rate $\left({ }^{\circ} \mathrm{C} / \mathrm{s}\right)$ \\
\hline Initial denaturation & 95 & 20 & $10\left(4.8^{\dagger}\right)$ & 95 & 20 & 4.8 \\
\hline Denaturation & 95 & 1 & $10\left(4.8^{\dagger}\right)$ & 95 & 1 & 4.8 \\
\hline Annealing extension ${ }^{*}$ & 60 & 10 & $10\left(4.8^{\dagger}\right)$ & 60 & 10 & 2.5 \\
\hline Cooling & 50 & 10 & $10\left(4.8^{+}\right)$ & 50 & 10 & 2.5 \\
\hline
\end{tabular}

Mastermix (Kapa Biosystems, Woburn, MA) (final concentration 1x; $1 \mu \mathrm{L}$ DNA or cDNA template, $5 \mu \mathrm{M}$ forward and reverse primers) in a final volume of $5 \mu \mathrm{L}$. The cycling conditions used for amplification of 18S rDNA are shown in Table 2 and employed the same ramp rate $\left(4.8^{\circ} \mathrm{C} / \mathrm{s}\right)$ on both machines. 18S rDNA amplification used $1 \mu \mathrm{L}$ of genomic DNA as template $(2.5 \mathrm{ng} / \mathrm{\mu l})$, either undiluted or serially diluted in PCR grade water. The same conditions were used for detection of miRNAs but with a reduced annealing/ extension temperature of $55^{\circ} \mathrm{C}$ and with ramp rates increased to $10^{\circ} \mathrm{C} / \mathrm{s}$ on the xxpress. Raw fluorescence data were analyzed by baseline normalization, and $\mathrm{C}_{\mathrm{a}}$ values were called in accordance with the data analysis software provided on each instrument (threshold set using the second derivative maximum algorithm on the LightCycler and manually on the xxpress).

RNA templates

An RNA oligonucleotide with the sequence of miR-21 and a poly $(A)$ tail (miRNA mimic) (Table 1) was reverse transcribed using $5 \mathrm{pmol}$ of oligodTRACE primer. The template was 

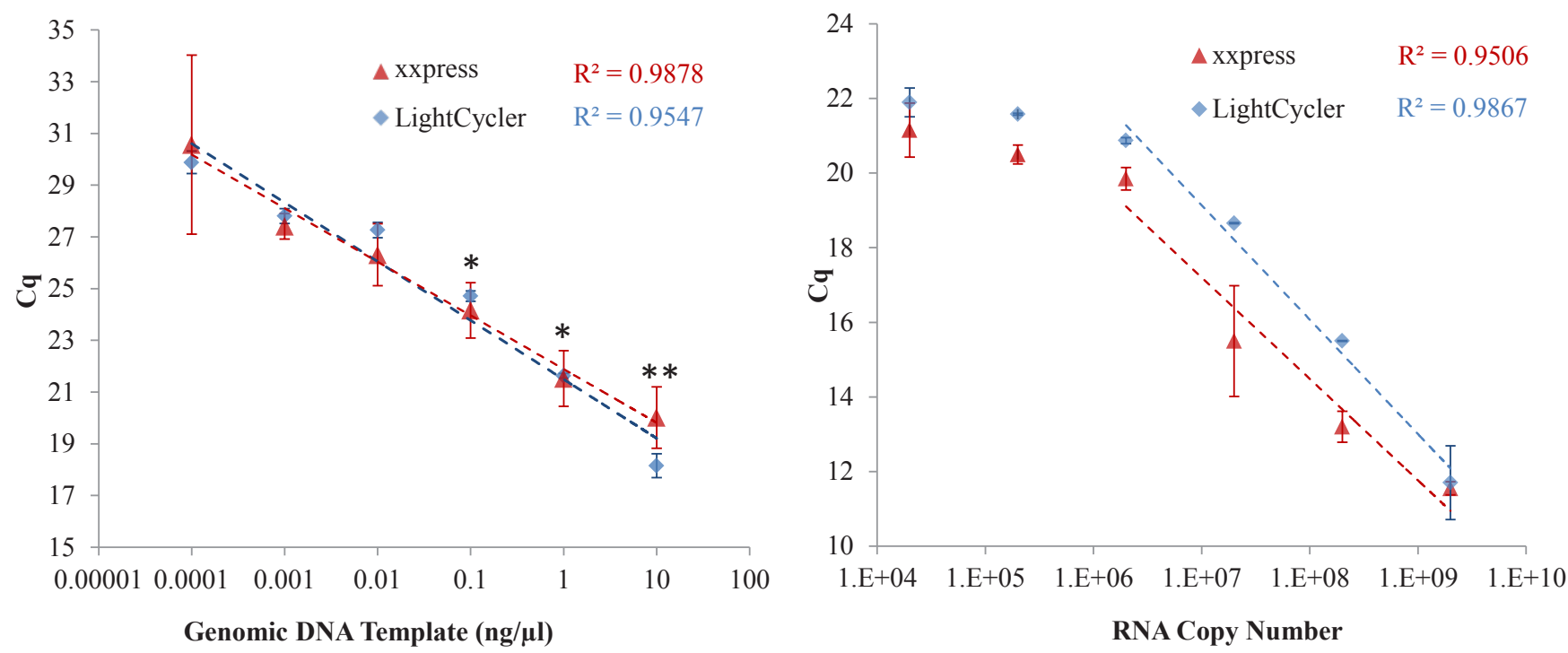

Figure 3. Standard curves generated by amplification of $18 \mathrm{~S}$ rDNA from genomic DNA and synthetic miR-21. (A) 18S rDNA standard curve analysis. Comparable dynamic range $0.1 \mathrm{pg}-10 \mathrm{ng}$ genomic DNA; amplified using the maximal ramp rate on each platform $\left(n=3\right.$ technical replicates) $\left({ }^{*} P<0.05\right.$, ${ }^{* *} P$ $<0.01$ ). (B) Synthetic miR-21 standard curve analysis. Comparable dynamic range: $10^{9}-10^{6}$ copies of template RNA; amplified using the maximal ramp rate on each platform ( $n=3$ technical replicates). For both data sets, melting curve analysis confirmed the presence of a single product (data not shown).

denatured at $50^{\circ} \mathrm{C}$ for $5 \mathrm{~min}$, then cooled to $4^{\circ} \mathrm{C}$ for addition of SuperScript III (Life Technologies, Paisley, UK) followed by incubation at $40^{\circ} \mathrm{C}$ for 60 min. Total RNA, including small RNAs, was extracted from $200 \mu \mathrm{L}$ human plasma using a miRNeasy serum/plasma kit according to the manufacturer's protocol (Qiagen, Crawley, UK) and polyadenylated for 1 h at $37^{\circ} \mathrm{C}$ in a $25 \mu \mathrm{L}$ reaction containing $2 \mathrm{U}$ poly(A) polymerase, $2.5 \mathrm{mM} \mathrm{MgCl}$, and $1 \mathrm{mM}$ ATP (Ambion, Austin, TX). A $5 \mu \mathrm{L}$ volume of polyadenylated RNA was then incubated at $65^{\circ} \mathrm{C}$ for $5 \mathrm{~min}$ with $0.5 \mu \mathrm{g}$ oligo(dT)-RACE primer and $1 \mu \mathrm{l} 10 \mathrm{mM}$ dNTPs in a reaction volume of $8 \mu \mathrm{L}$. Following addition of $200 \mathrm{U}$ SuperScript III, $1 \cup$ RNaseOut, $1 \mu \mathrm{L}$ $0.1 \mathrm{M}$ DTT, and $4 \mu \mathrm{L} 5 \times \mathrm{RT}$ buffer, the reaction was incubated at $50^{\circ} \mathrm{C}$ for 1 $\mathrm{h}$ followed by $70^{\circ} \mathrm{C}$ for $15 \mathrm{~min}$.

\section{Ethics}

This study was conducted according to the guidelines laid down in the Declaration of Helsinki, and all procedures involving human participants/patients were approved by the Research Ethics Committee of the School of Medicine and Dentistry, Queen's University Belfast (Ref:11/05v3). Written informed consent was obtained from all participants.

\section{Results and discussion}

To assess consistency across the plate, a single mastermix for amplification of a $155 \mathrm{bp}$ amplicon from 18S rDNA was aliquoted into 48 wells distributed evenly across each type of plate (Supplementary Figure S1). Amplification was performed using the same cycling conditions on both systems (Table 2). The mean $\mathrm{C}_{0}$ values were very similar for the xxpress $(20.01 \pm 0.47 \mathrm{SD})$ and the LightCycler $(19.87 \pm 0.04 \mathrm{SD})$, although the LightCycler was significantly lower (t-test, $P$ $=0.035)$. However, the xxpress exhibited significantly greater variability than the LightCycler, which generated extremely consistent $C_{a}$ values ( $F$-test, $P=2.4 \times$ 10-25) (Figure 2). Although the performance of the xxpress was less robust, it could still be used in situations requiring rapid detection of large variations in expression between samples (with the inclusion of appropriate replicates). The spread in $\mathrm{C}_{\mathrm{q}}$ values on the xxpress was in part linked to the position of the sample on the xxplate, with adjacent wells tending to vary from the mean $\mathrm{C}_{0}$ value in the same direction. The greater variation observed with the xxpress may not be due to poorer temperature control across the plate, but could be related to the optical system, which is amenable to improvement in subsequent versions. To compare the sensitivity of the two platforms, highly abundant 18S rDNA was amplified from a dilution series of genomic DNA. The mean $\mathrm{C}_{\mathrm{q}}$ values at each template concentration were not significantly different between platforms (t-test, $P>0.05)$. In both cases, the $C_{q}$ values were highly correlated with template concentration (LightCycler, $R^{2}$ $=0.9547$; xxpress, $R^{2}=0.9878$ ), although again there was a significantly greater variation in $\mathrm{C}_{\mathrm{q}}$ values for replicates in the xxpress at several concentrations (Figure 3A).

The method described by Shi and Chiang (14) was then used to detect miRNAs. Total RNA, including miRNAs, is polyadenylated and then reversetranscribed with a poly $(\mathrm{T})$ adapter into cDNAs. These are subsequently amplified using an miRNA-specific forward primer and a sequence complementary to the poly $(T)$ adapter as the reverse primer. Initially, we tested the ability of each platform to detect an miRNA mimic synthesized with a poly(A) tail already incorporated (thereby removing any variability associated with the polyadenylation reaction). The limit of detection, as estimated from the linear portion of the standard curve, was approximately $1 \times 10^{6}$ RNA template molecules for 


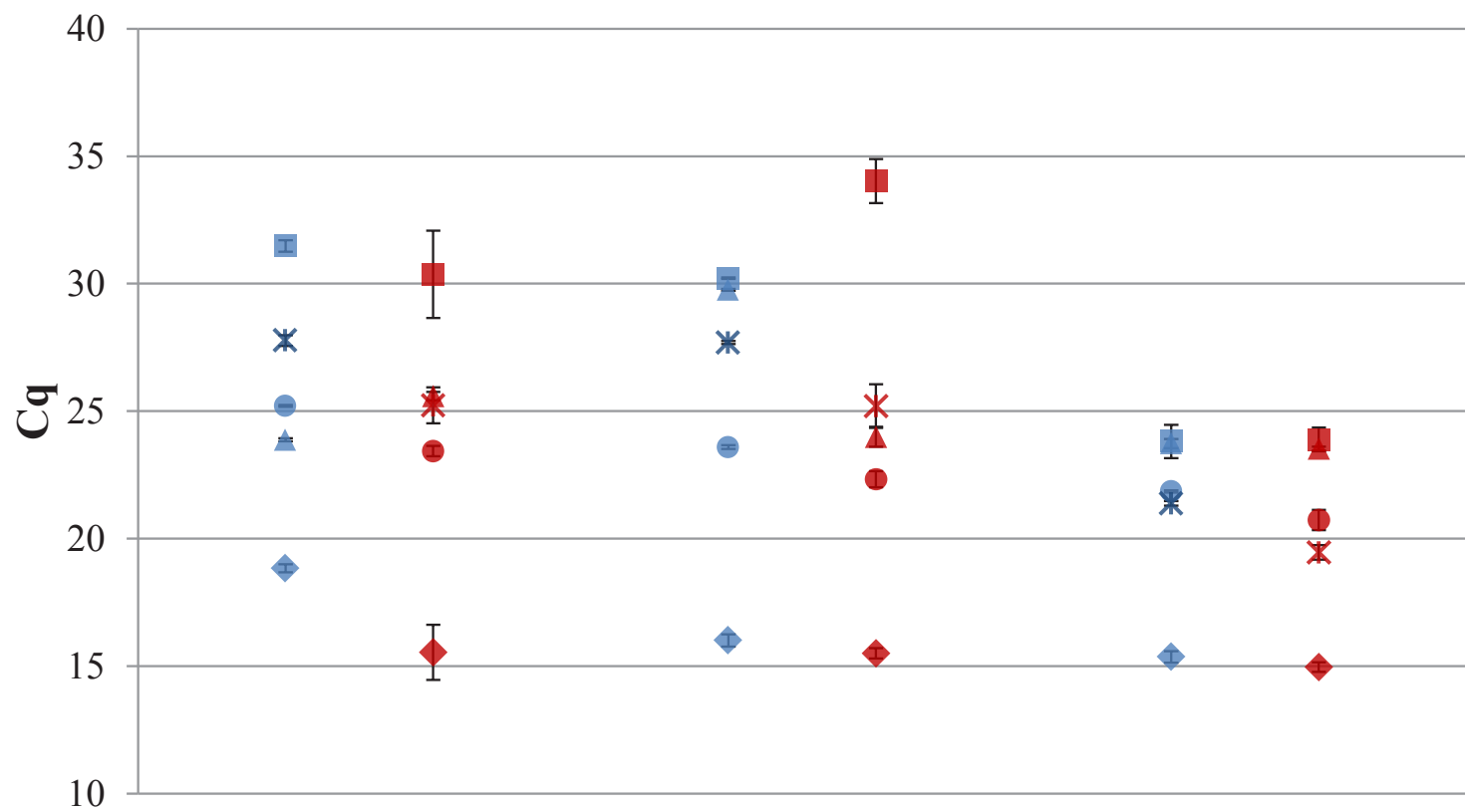

xxpress

LightCycle

- miR-21

miR-22

$\triangle$ miR-126

- miR-486

*Y-RNA-3p

Plasma 1

Plasma 2

Plasma 3

Figure 4. Detection of microRNAs from human plasma samples by fast and standard PCR analysis. C values for 4 microRNAs (miR-21, miR-22, miR-126, miR-486) and Y-RNA (Y-RNA-3p) in plasma samples from 3 individuals ( $n=3$ technical replicates). The relative levels of expression detected by both systems were comparable. Gel and melting curve analysis was carried out to confirm amplification of one product (data not shown).

Sometimes it takes more than one slice to make a great sandwich.

\section{The SageELF ${ }^{\mathrm{TM}}$ from Sage Science.}

\section{Collect multiple slices from a single sample.}

Automated Gel Purification for:

- Next Gen Sequencing

- Protein Mass Spec
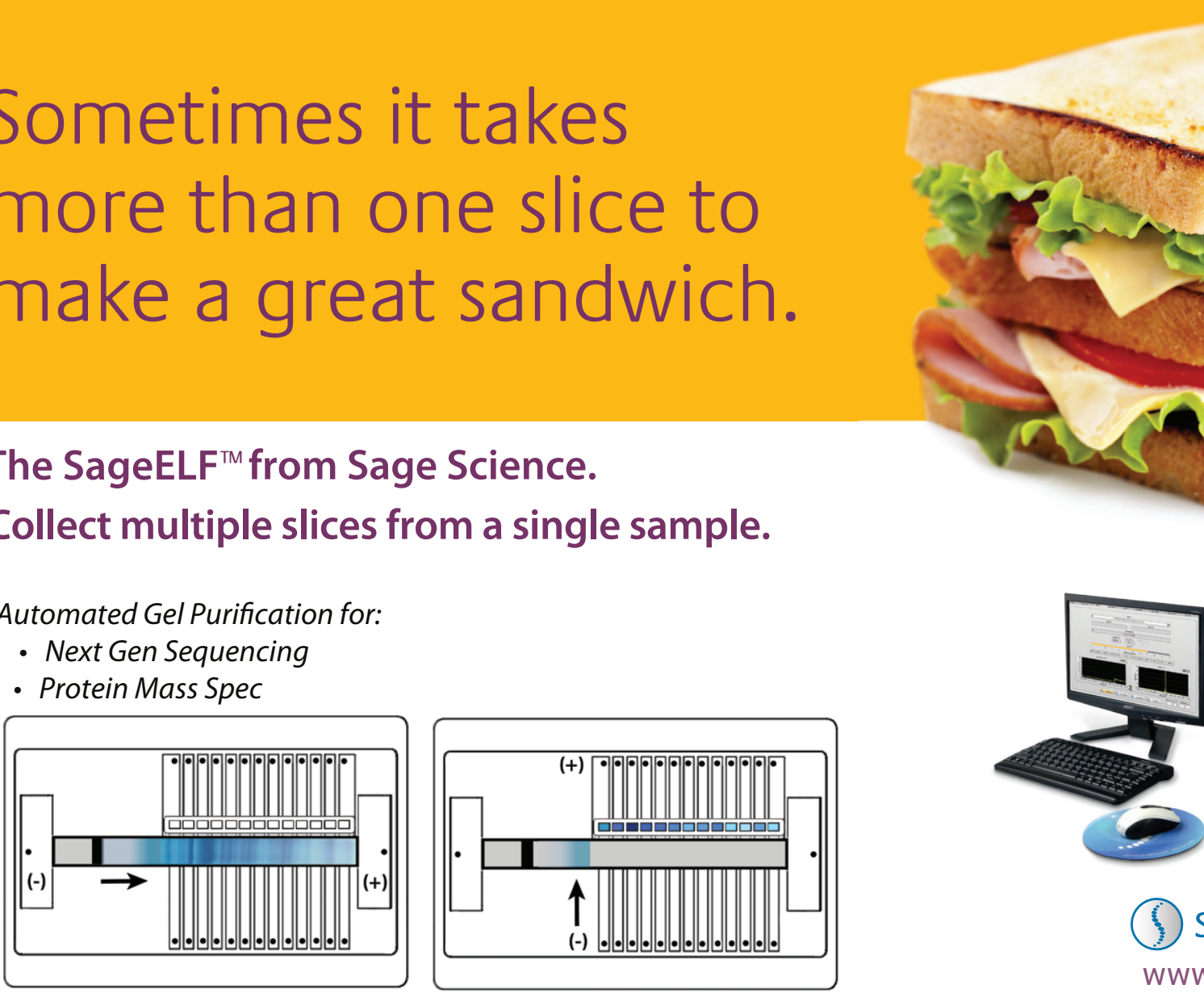
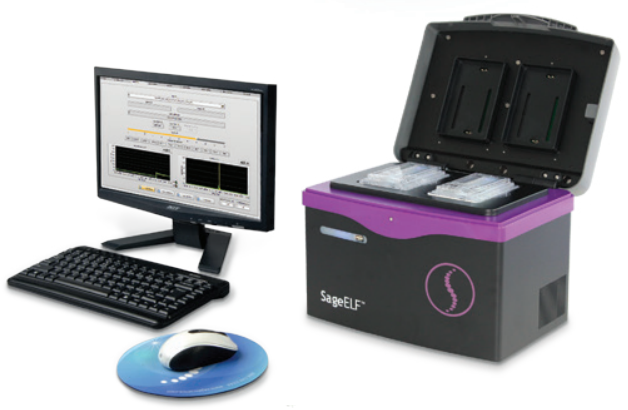

() sage science www.sagescience.com 
both systems (Figure 3B). Given the high concentrations of specific template miRNA used in this assay, a better signal may be obtained using cell-derived RNA as template (15).

Our ultimate aim is the rapid detection of miRNAs from blood as biomarkers of cancer and other diseases. As a step toward this goal, we measured the expression of several miRNAs in RNA extracted from three human plasma samples: miR-21, an "oncomir" that plays a role in many types of cancer (16-18) and heart disease (19) and has been reported to be elevated in blood from cancer patients $(20,21)$; miR-22, which is also differentially expressed in various types of cancer (22); miR-126, which is involved in regulation of angiogenesis (23); and miR-486, which is down-regulated in lung cancer and proposed as a biomarker for detection of lung cancer in plasma samples $(24,25)$. The results of quantification of these 4 miRNAs following 40 cycles of PCR, completed in less than 10 min with the xxpress compared with 40 min using the LightCycler, are presented in Figure 4. The relative abundances of the miRNAs detected by both systems were similar, albeit with greater variation between technical replicates on the xxpress. MiR-21 was the most abundant (lowest $\mathrm{C}_{\mathrm{a}}$ ) miRNA and miR-22 the least abundant (highest $\mathrm{C}_{\mathrm{q}}$ ) for all samples on both platforms. Significant differences between platforms in $C$ values were observed for many miRNAs amplified from the same sample (e.g., miR-126 in plasma sample 2; $P<0.001)$. This is likely due to the combined effects of differences in the sample temperatures achieved during cycling, the detection systems, and the performance of the individual assays under different conditions. Another class of small RNA, a $Y$ RNA fragment (hY4-3p) recently shown to be present in plasma (26-28) was detected consistently by both platforms $\left(\triangle \mathrm{C}_{\mathrm{a}}\right.$ between the 2 platforms across 3 plasma samples was $2.3 \pm 0.3 \mathrm{SD}$ ) and could potentially provide a stable reference gene.

Faster PCR can be achieved by reducing hold times, and although this can reduce sensitivity and increase variability (29), enzymes are now available from a range of manufacturers that perform well in fast PCR. All data reported were generated using SYBR fast qPCR Mastermix (Kapa Biosystems), but $18 S$ rDNA failed to amplify using Quantitect (Qiagen) on the xxpress, so users should be aware that rigorous optimization of different mastermixes is required for fast $\mathrm{PCR}$. To facilitate development of rapid assays incorporating fast PCR, pre-processing steps must be minimized. There is therefore a growing need to develop enzymes resistant to contaminants and inhibitors present in a range of biological sample materials, such as soil, water and blood (30).

Although many prototype rapid PCR systems have been reported with extremely fast ramp rates and miniaturization $(5,6,31)$, very few proceed to commercial release. Therefore, the xxpress rapid thermal cycler, particularly given its standard block-based design, offers a unique opportunity for the wider molecular research community to adopt fast PCR. The potential applications of fast PCR in situations that require a rapid diagnosis [e.g., involvement of cardiac disease in dyspnoea (32) or chest pain] make this an exciting and rapidly expanding area of PCR devel-

\section{IIlluminating Solutions For Your Life Science Research!}

Life science and laboratory research professionals depend on high-quality Spectroline ${ }^{\circledR}$ ultraviolet products to make their work faster and easier. Our products are built to exacting standards, ensuring years of reliable, trouble-free service.

\section{UV Crosslinkers}

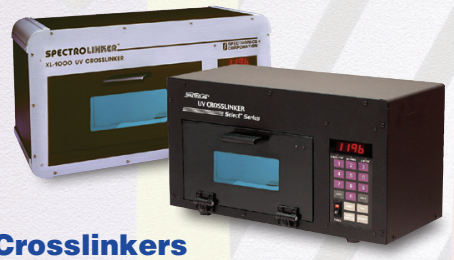

Provide accurate, error-free and super-fast

membrane crosslinking and sample UV dosing.

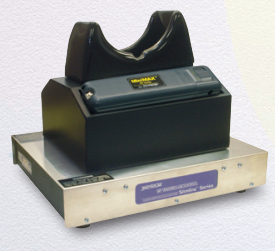

UV-Protective Eye and Face Wear Specially designed to protect users against unwanted UV exposure.

MiniMAX ${ }^{\mathrm{TM}}$ UV Viewing Station Space-saving mini-cabinet and UV lamp combination makes the perfect portable UV viewing station. Light weight and compact. CORPORATION wWW.spectroline.com

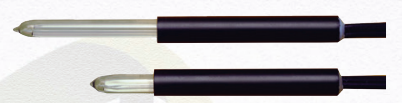

Miniature UV Pencil Lamps Double-bore quartz lamps used for wavelength calibration and emission. Custom lamps for OEM applications also available.

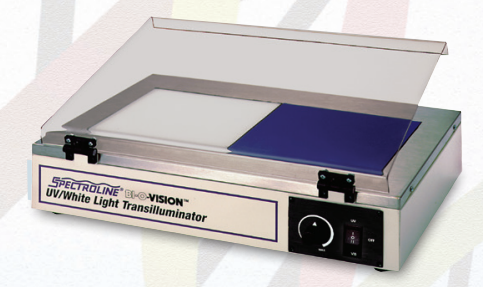

UV Transilluminators

Deliver superior irradiance uniformity and UV intensity for all viewing and photodocumentation needs.

Buy Direct From Us and Save!

Call 1-800-274-8888 (516-333-4840 outside USA) or email us at sales@spectroline.com and mention Code B315

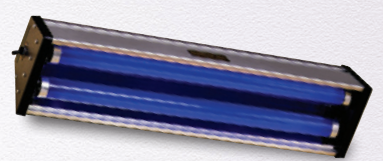

UV Bench and Display Lamps Lamps with various wattages and UV wavelengths provide both high intensity and wide area of light coverage.

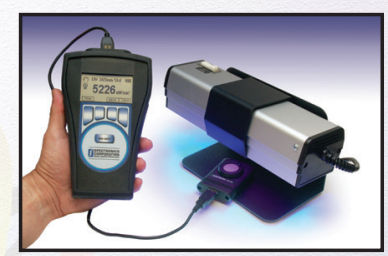

AccuMAXTM Digital Meters Advanced microprocessor-controlled units with software-driven functions. Ensure maximum accuracy and reliability in measurement of UV and visible light sources.

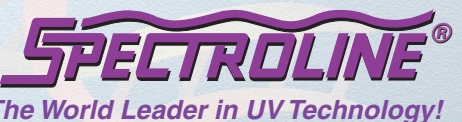


opment. If protocols can be developed to enable reverse transcription and PCR directly from patient samples (30), this platform has the potential to make pointof-care diagnostics based upon circulating microRNAs a reality.

\section{Author contributions}

W.J.A. performed and analyzed the experiments and assisted with manuscript preparation. E.B. and M.D. performed experiments with plasma and assisted with manuscript preparation. R.E.H. provided plasma samples and assisted with manuscript preparation. D.A.S. conceived the study, analyzed the data, and wrote the manuscript.

\section{Acknowledgments}

This work was supported by grants from the Department of Education and Learning (DEL), Invest Northern Ireland (INI), the European Regional Development Fund, and Fight For Sight.

\section{Competing interests}

The authors declare no competing interests.

\section{References}

1. Laurin, N. and C. Frégeau. 2012. Optimization and validation of a fast amplification protocol for AmpFISTR $®$ Profiler Plus $\AA$ for rapid forensic human identification. Forensic Sci. Int. Genet. 6:47-57.

2. Bahlmann, S., S. Hughes-Stamm, and D. Gangitano. 2014. Development and evaluation of a rapid PCR method for the PowerPlex®S5 system for forensic DNA profiling. Leg Med (Tokyo). 16:227-233.

3. Wheeler, E.K., C.A. Hara, J. Frank, J. Deotte, S.B. Hall, W. Benett, C. Spadaccini, and N.R. Beer. 2011. Under-three minute PCR: probing the limits of fast amplification. Analyst. 136:3707-3712.

4. Neuzil, P., C. Zhang, J. Pipper, S. Oh, and L. Zhuo. 2006. Ultra fast miniaturized real-time PCR: 40 cycles in less than six minutes. Nucleic Acids Res. 34:e77.

5. Hashimoto, M., P.-C. Chen, M.W. Mitchell, D.E. Nikitopoulos, S.A. Soper, and M.C. Murphy. 2004. Rapid PCR in a continuous flow device. Lab Chip 4:638-645.

6. Farrar, J.S. and C.T. Wittwer. 2015. Extreme PCR: Efficient and Specific DNA Amplification in 15-60 Seconds. Clin. Chem. 61:145-153.

7. Wittwer, C.T., K.M. Ririe, R.V. Andrew, D.A. David, R.A. Gundry, and U.J. Balis. 1997. The LightCycler: a microvolume multisample fluorimeter with rapid temperature control. Biotechniques 22:176-181.

8. Mitchell, P.S., R.K. Parkin, E.M. Kroh, B.R. Fritz, S.K. Wyman, E.L. Pogosova-
Agadjanyan, A. Peterson, J. Noteboom, et al. 2008. Circulating microRNAs as stable blood-based markers for cancer detection. Proc. Natl. Acad. Sci. USA 105:10513-10518.

9. Chen, X., Y. Ba, L. Ma, X. Cai, Y. Yin, K. Wang, J. Guo, Y. Zhang, et al. 2008. Characterization of microRNAs in serum: a novel class of biomarkers for diagnosis of cancer and other diseases. Cell Res. 18:9971006.

10. Creemers, E.E., A.J. Tijsen, and Y.M. Pinto. 2012. Circulating microRNAs: novel biomarkers and extracellular communicators in cardiovascular disease? Circ. Res. 110:483-495

11. Weiland, M., X.-H. Gao, L. Zhou, and Q.-S. Mi. 2012. Small RNAs have a large impact: circulating microRNAs as biomarkers for human diseases. RNA Biol. 9:850-859.

12. Leidinger, P., C. Backes, S. Deutscher, K. Schmitt, S.C. Mueller, K. Frese, J. Haas, K. Ruprecht, et al. 2013. A blood based 12-miRNA signature of Alzheimer disease patients. Genome Biol. 14:R78.

13. Deddens, J.C., J.M. Colijn, M.I.F.J. Oerlemans, G. Pasterkamp, S.A. Chamuleau, P.A. Doevendans, and J.P.G. Sluijter. 2013. Circulating microRNAs as novel biomarkers for the early diagnosis of acute coronary syndrome. J. Cardiovasc. Transl. Res. 6:884-898.

14. Shi, R. and V.L. Chiang. 2005. Facile means for quantifying microRNA expression by real-time PCR. Biotechniques 39:519-525.

15. Levesque-Sergerie, J.-P., M. Duquette, C. Thibault, L. Delbecchi, and N. Bissonnette. 2007. Detection limits of several commercial reverse transcriptase enzymes: impact on the low- and high-abundance transcript levels assessed by quantitative RT-PCR. BMC Mol. Biol. 8:93.

16. Selcuklu, S.D., M.T.A. Donoghue, and C. Spillane. 2009. miR-21 as a key regulator of oncogenic processes. Biochem. Soc. Trans. 37:918-925.

17. Iorio, M.V., M. Ferracin, C.-G. Liu, A. Veronese, R. Spizzo, S. Sabbioni, E. Magri, M. Pedriali, et al. 2005. MicroRNA Gene Expression Deregulation in Human Breast Cancer. Cancer Res. 65:7065-7070.

18. Volinia, S., G.A. Calin, C.-G. Liu, S. Ambs, A. Cimmino, F. Petrocca, R. Visone, M. lorio, et al. 2006. A microRNA expression signature of human solid tumors defines cancer gene targets. Proc. Natl. Acad. Sci. USA 103:2257-2261.

19. Thum, T., C. Gross, J. Fiedler, T. Fischer, S. Kissler, M. Bussen, P. Galuppo, S. Just, et al. 2008. MicroRNA-21 contributes to myocardial disease by stimulating MAP kinase signalling in fibroblasts. Nature 456:980-984

20. Asaga, S., C. Kuo, T. Nguyen, M. Terpenning, A.E. Giuliano, and D.S.B. Hoon. 2011. Direct serum assay for microRNA-21 concentrations in early and advanced breast cancer. Clin. Chem. 57:84-91.

21. Xu, J., C. Wu, X. Che, L. Wang, D. Yu, T. Zhang, L. Huang, H. Li, et al. 2011. Circulating MicroRNAs, miR-21, miR-122, and miR-223, in patients with hepatocel- lular carcinoma or chronic hepatitis. Mol. Carcinog. 50:136-142

22. Zhang, J., Y. Yang, T. Yang, Y. Liu, A. Li, S. Fu, M. Wu, Z. Pan, and W. Zhou. 2010. microRNA-22, downregulated in hepatocellular carcinoma and correlated with prognosis, suppresses cell proliferation and tumourigenicity. Br. J. Cancer 103:1215-1220.

23. Wang, S., A.B. Aurora, B.A. Johnson, X. Qi, J. McAnally, J.A. Hill, J.A. Richardson, R. Bassel-Duby, and E.N. OIson. 2008. The endothelial-specific microRNA miR-126 governs vascular integrity and angiogenesis. Dev. Cell 15:261-271.

24. Yu, L., N.W. Todd, L. Xing, Y. Xie, H. Zhang, Z. Liu, H. Fang, J. Zhang, et al. 2010. Early detection of lung adenocarcinoma in sputum by a panel of microRNA markers. Int. J. Cancer 127:2870-2878.

25. Xing, L., N.W. Todd, L. Yu, H. Fang, and F. Jiang. 2010. Early detection of squamous cell lung cancer in sputum by a panel of microRNA markers. Mod. Pathol. 23:1157-1164.

26. Beatty, M., J. Guduric-Fuchs, E. Brown, S. Bridgett, U. Chakravarthy, R.E. Hogg, and D.A. Simpson. 2014. Small RNAs from plants, bacteria and fungi within the order Hypocreales are ubiquitous in human plasma. BMC Genomics 15:933.

27. Dhahbi, J.M., S.R. Spindler, H. Atamna, D. Boffelli, P. Mote, and D.I.K. Martin. 2013. 5'-YRNA fragments derived by processing of transcripts from specific YRNA genes and pseudogenes are abundant in human serum and plasma. Physiol. Genomics 45:990-998.

28. Dhahbi, J.M., S.R. Spindler, H. Atamna D. Boffelli, and D.I. Martin. 2014. Deep Sequencing of Serum Small RNAs Identifies Patterns of 5' tRNA Half and YRNA Fragment Expression Associated with Breast Cancer. Biomark Cancer. 6:37-47

29. Hilscher, C., W. Vahrson, and D.P. Dittmer. 2005. Faster quantitative real-time PCR protocols may lose sensitivity and show increased variability. Nucleic Acids Res. 33:e182.

30. Kermekchiev, M.B., L.I. Kirilova, E.E. Vail, and W.M. Barnes. 2009. Mutants of Taq DNA polymerase resistant to PCR inhibitors allow DNA amplification from whole blood and crude soil samples. Nucleic Acids Res. 37:e40.

31. Salm, E., Y. Zhong, B. Reddy, C. DuarteGuevara, V. Swaminathan, Y.-S. Liu, and R. Bashir. 2014. Electrical detection of nucleic acid amplification using an on-chip quasireference electrode and a PVC REFET. Anal. Chem. 86:6968-6975.

32. Tijsen, A.J., E.E. Creemers, P.D. Moerland, L.J. de Windt, A.C. van der Wal, W.E. Kok, and Y.M. Pinto. 2010. MiR423-5p as a circulating biomarker for heart failure. Circ. Res. 106:1035-1039.

Received 23 January 2015; accepted 06 March 2015

Address correspondence to David Arthur Simpson, Centre for Experimental Medicine, Queen's University Belfast, Institute of Clinical Science, Royal Victoria Hospital, Belfast, Northern Ireland, United Kingdom. E-mail: David.Simpson@qub.ac.uk

To purchase reprints of this article, contact: biotechniques@fosterprinting.com 